\title{
Supporting information for: Stretchable All-Dielectric Metasurfaces with Polarization-Insensitive and Full-Spectrum Response
}

Chen Zhang ${ }^{1}$, Jixiang Jing ${ }^{1}$, Yunkai Wu ${ }^{1}$, Yubin Fan ${ }^{1}$, Wenhong Yang ${ }^{1}$, Shuai Wang ${ }^{1}$, Qinghai Song ${ }^{1,2, *}$, Shumin Xiao ${ }^{1,2, \#}$

1. State Key Laboratory on Tunable laser Technology, Ministry of Industry and Information Technology Key Lab of Micro-Nano Optoelectronic Information System, Shenzhen Graduate School, Harbin Institute of Technology, Shenzhen, 518055, P. R. China.

2. Collaborative Innovation Center of Extreme Optics, Shanxi University, Taiyuan, 030006, Shanxi, P. R. China.

${ }^{\#}$ shumin.xiao@hit.edu.cn; * qinghai.song@hit.edu.cn

The simulation unit cell is schematized in Figure S1. The $\mathrm{TiO}_{2}$ nanoblocks are located on top of PDMS with refractive index of 1.4 and embedded in surrounding media with different refractive index. The reflected spectra of the metasurface were taken with the refractive index of surrounding media between nanoblocks varying from 1.0 to $1.2,1.4$ and 1.6 . 


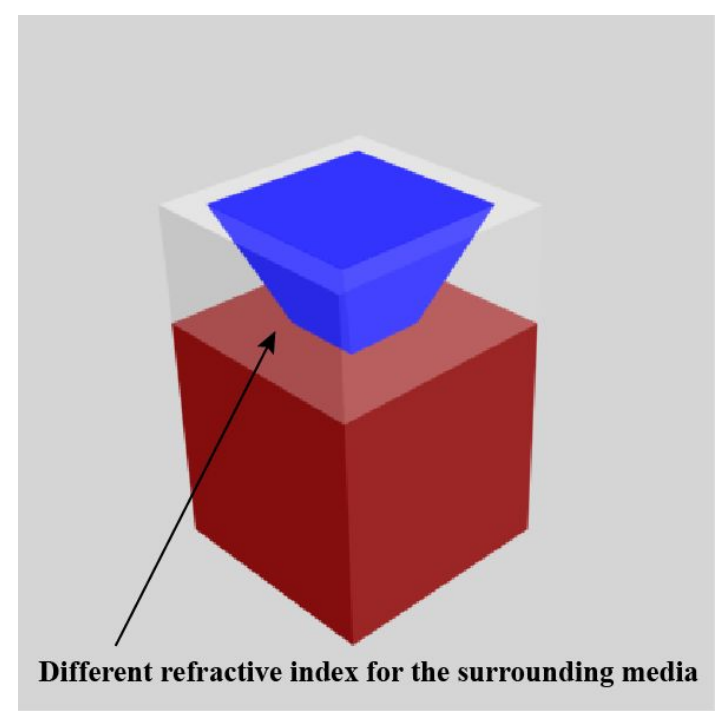

Figure S1. The schematic of the simulated unit cell

Commercially available finite element method (FEM)-based software, COMSOL Multiphysics 5.4, was used to perform all the numerical calculations. Periodic boundary conditions were used to simulate the periodic array of the nanoblocks. $\mathrm{TiO}_{2}$ antenna array was settled on a PDMS substrate, with the optical constant of $\mathrm{TiO}_{2}$ determined from spectroscopic ellipsometry measurement. Here the period p, nanoblock width w, and the height are $300 \mathrm{~nm}, 230 \mathrm{~nm}$, and $200 \mathrm{~nm}$, respectively.

The simulation reflection result for $\mathrm{x}$-polarization incident light is shown in Figure S2. With the same stretching condition along x-direction, the x-polarization wave shall experience the similar processes as the lattice size $p_{\mathrm{x}}$ increases, i.e. the MD resonance red-shifts, whereas the ED resonance blue-shifts for metasurface with surrounding media with refractive index increasing from 1.0, 1.2, 1.4 to 1.6. 

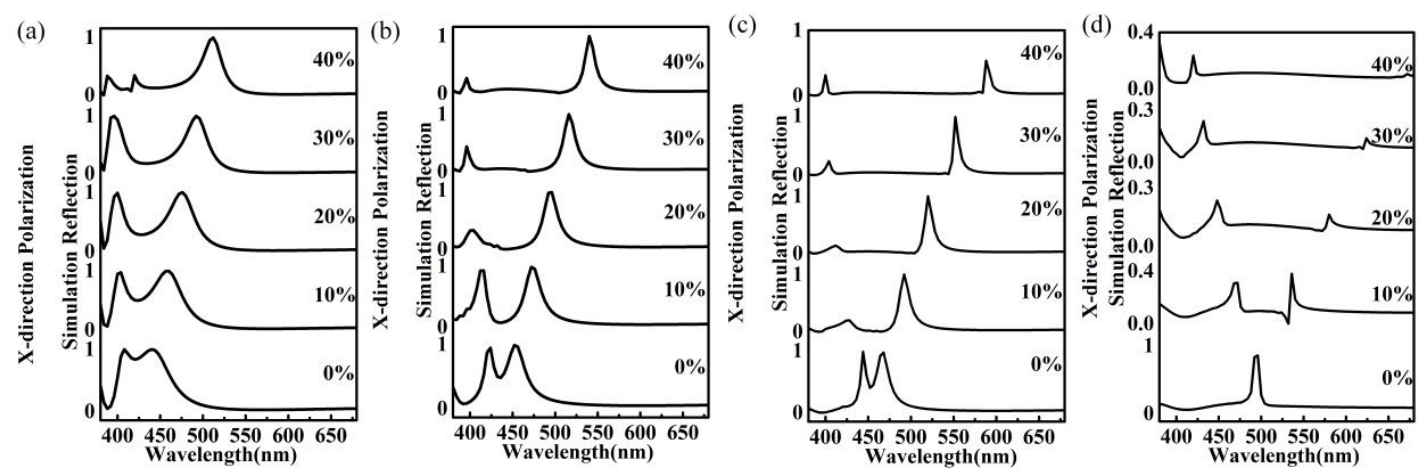

Figure S2. The numerically calculated reflection spectrum. (a) The refractive index is

1.0, the reflection spectrum with different Stretch percentage value for x-polarization incident light. (b) (c) and (d) are the corresponding reflection spectrums with refractive index increasing from 1.2, 1.4 to 1.6.

By increasing the effective refractive index, the refractive index contrast is decreased. With $\mathrm{TiO}_{2}$ metasurface embedded in surrounding media with different refractive index for $\mathrm{x}$-polarization incident light from the glass, the MD resonance corresponding electric field is mostly circulating within etch nanoblock, and ED resonance distributes outside the nanoblock and propagates between neighboring nanoblocks. As a result, the near-field interaction of MD resonance is reduced and caused the red-shift of MD resonance. The corresponding ED resonance generated the near-field mutual interaction between adjacent nanoblocks, the resonant frequency increases and the ED resonance shift to shorter wavelength. With the refractive index changes in Figure $\mathbf{S 3}$ (a), the peak position will shift significantly. The corresponding field diagram is shown in Figure S3 (b). As shown in the Figure S3 (c) and Figure S3 (d), this mechanism of MD and ED shift with refractive index is also suitable for the sample stretched $20 \%$. 

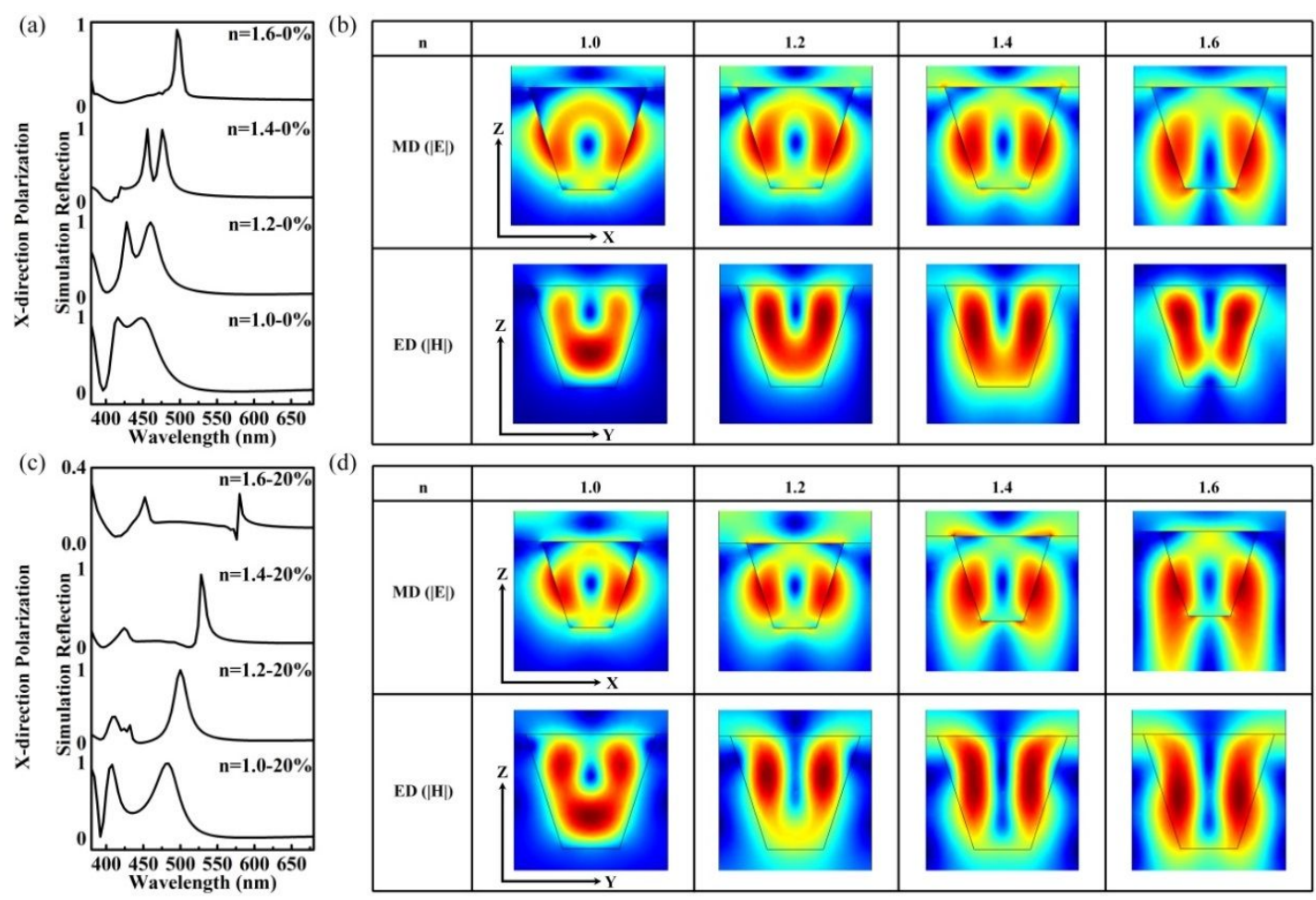

Figure S3. The field patterns of $\mathrm{TiO}_{2}$ metasurface under different refractive index. (a)

The wavelength shift as the refractive index of surrounding medium changes. (b) The field distribution of MD resonance and ED resonance in x-polarization. (c) and (d) are respectively reflection spectrum and field patterns with $20 \%$ strain.

The simulation reflection result for y-polarization incident light is shown in Figure S4. With the same stretching condition along x-direction, the y-polarization wave will experience much more complicated processes as the lattice size $p_{\mathrm{x}}$ increases. 

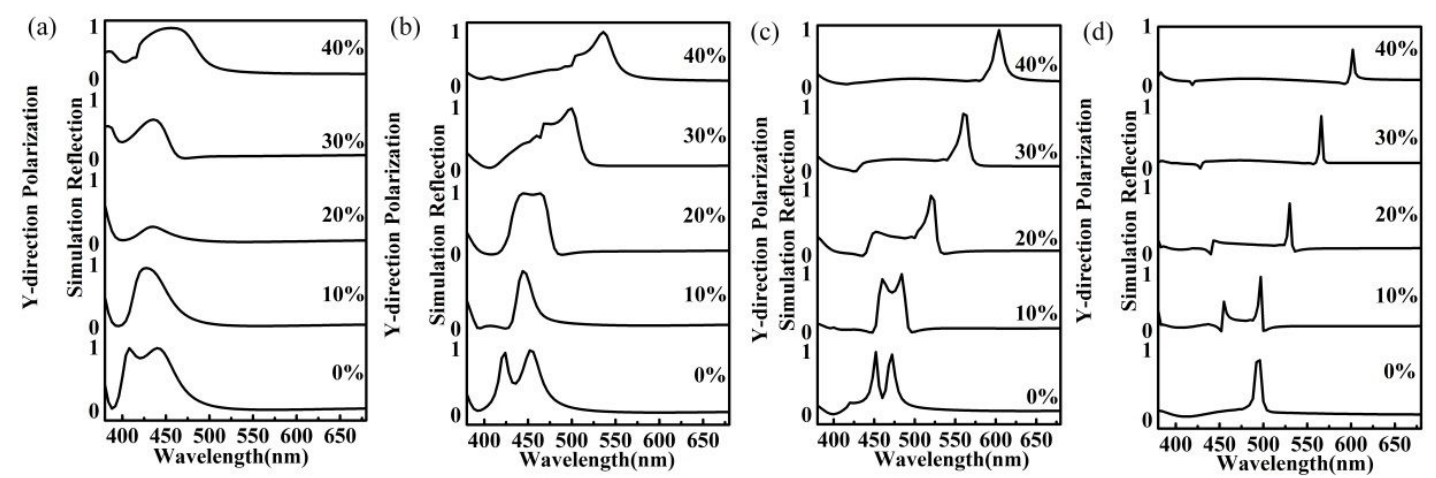

Figure S4. The numerically calculated reflection spectrum. (a) The refractive index is 1.0, the reflection spectrum with different Stretch percentage value for y-polarization incident light. (b) (c) and (d) are the corresponding reflection spectrums with refractive index increasing from 1.2, 1.4 to 1.6.
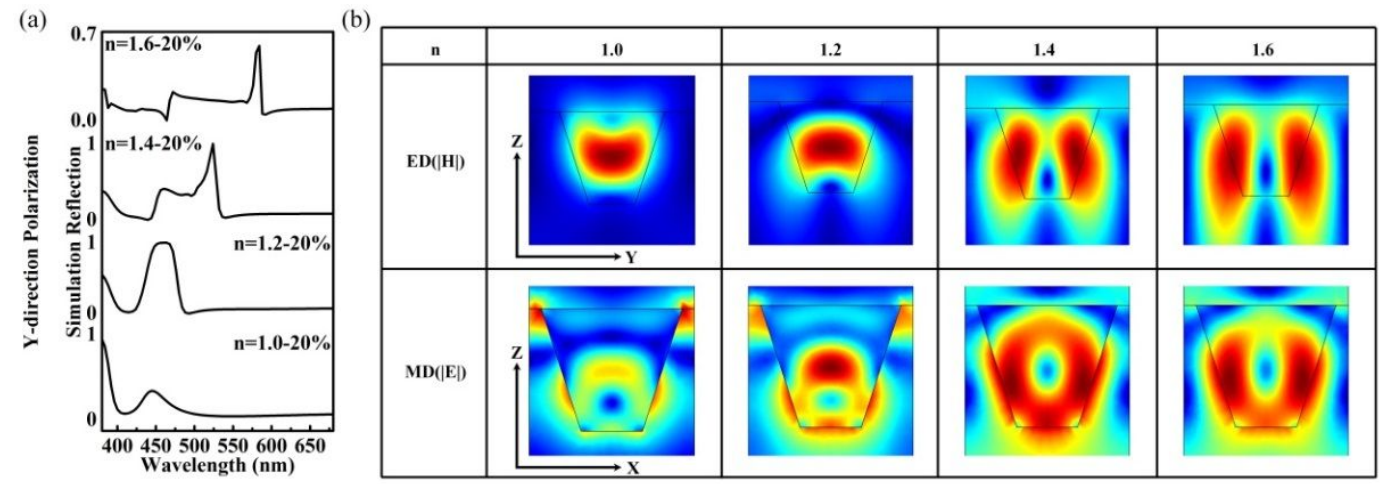

Figure S5. The field patterns of $\mathrm{TiO}_{2}$ metasurface under different refractive index with $20 \%$ strain. (a) The wavelength shift as the refractive index of surrounding medium changes. (b) The field distribution of MD resonance and ED resonance in y-polarization with $20 \%$ strain.

According to the field distribution in Figure S5, it can be analyzed that with the same stretching condition along x-direction, the y-polarization shall experience the reversed processes as the refractive index increases, i.e. the MD resonance blue-shifts 
with the increase of refractive index, whereas the ED resonance red-shifts with refractive index increasing from 1.2, 1.4 to 1.6.

The schematic of the simulation is shown in Figure S6 and unit cell with different filling factor of the surrounding media. The $\mathrm{TiO}_{2}$ metasurface covered in PDMS from $0 \%, 25 \%$ to $100 \%$.

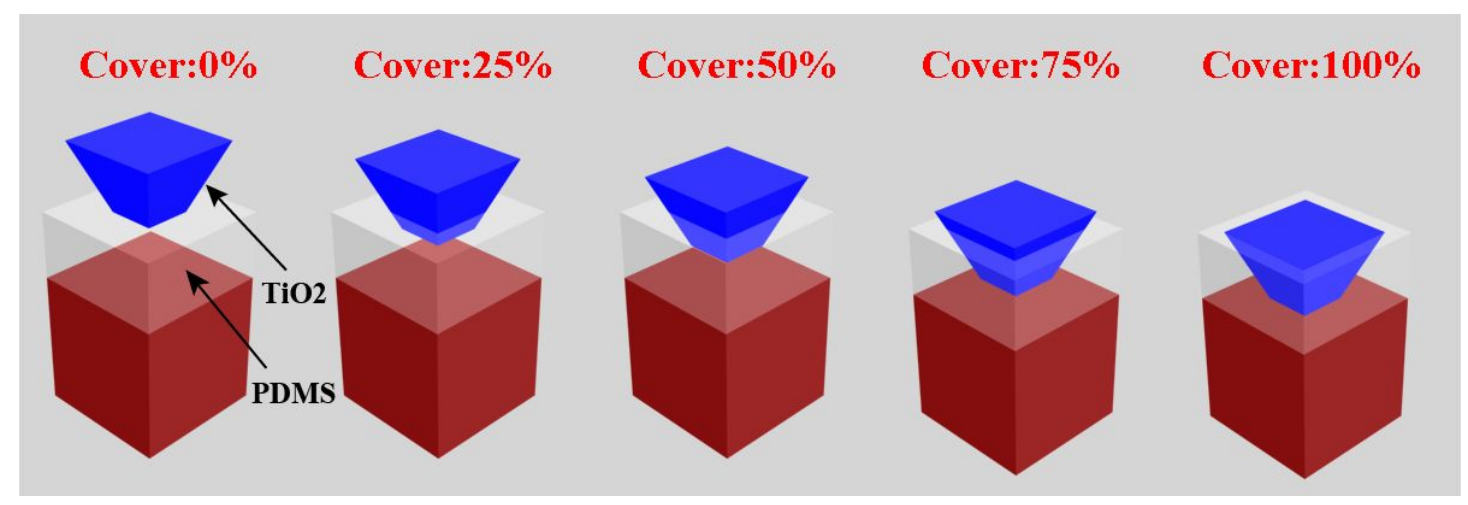

Figure S6. The schematics of the simulated unit cell with different filling factor of the surrounding media.

The reflection spectrum of the metasurface with different filling factors of PDMS inside the gap is demonstrated in Figure S7 and Figure S8. Here the period $p$, nanoblock width $w$, and the height are $300 \mathrm{~nm}, 230 \mathrm{~nm}$, and $200 \mathrm{~nm}$, respectively. With a filling factor, the reflection spectrum corresponds to different stretching amounts. And the strain is from $0 \%, 10 \%$ to $40 \%$. 


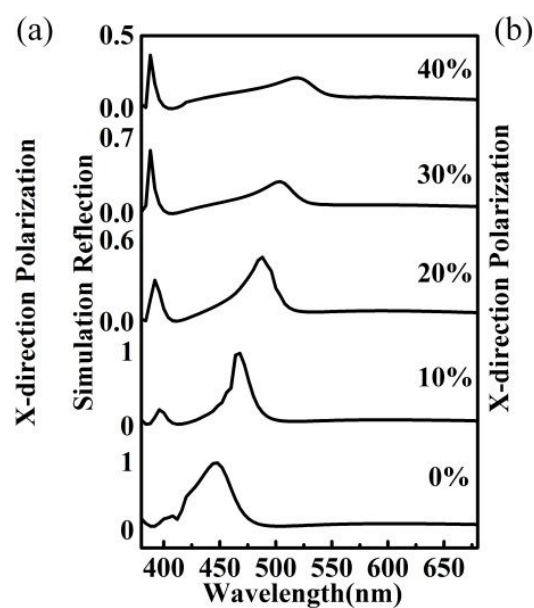

(d)

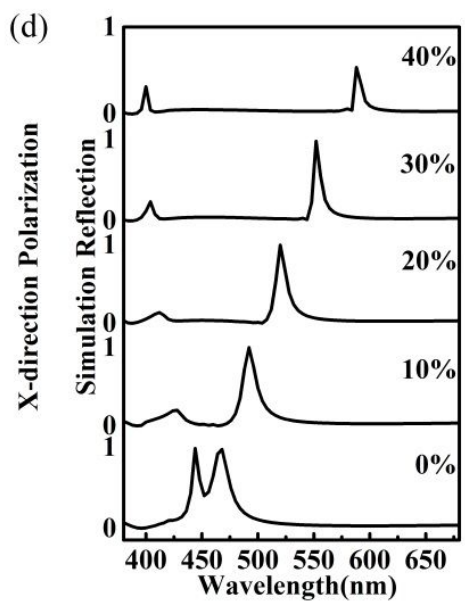

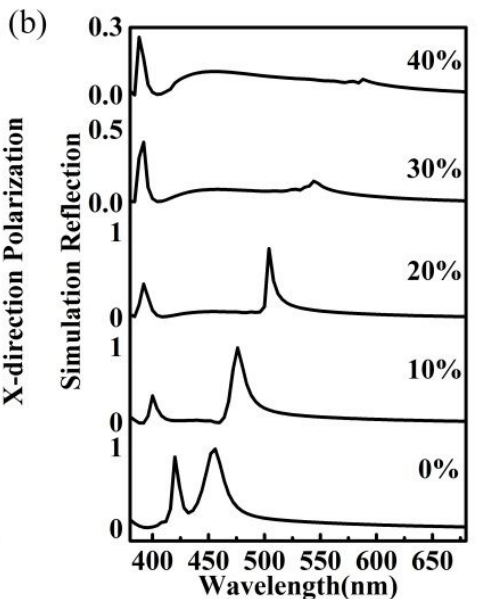
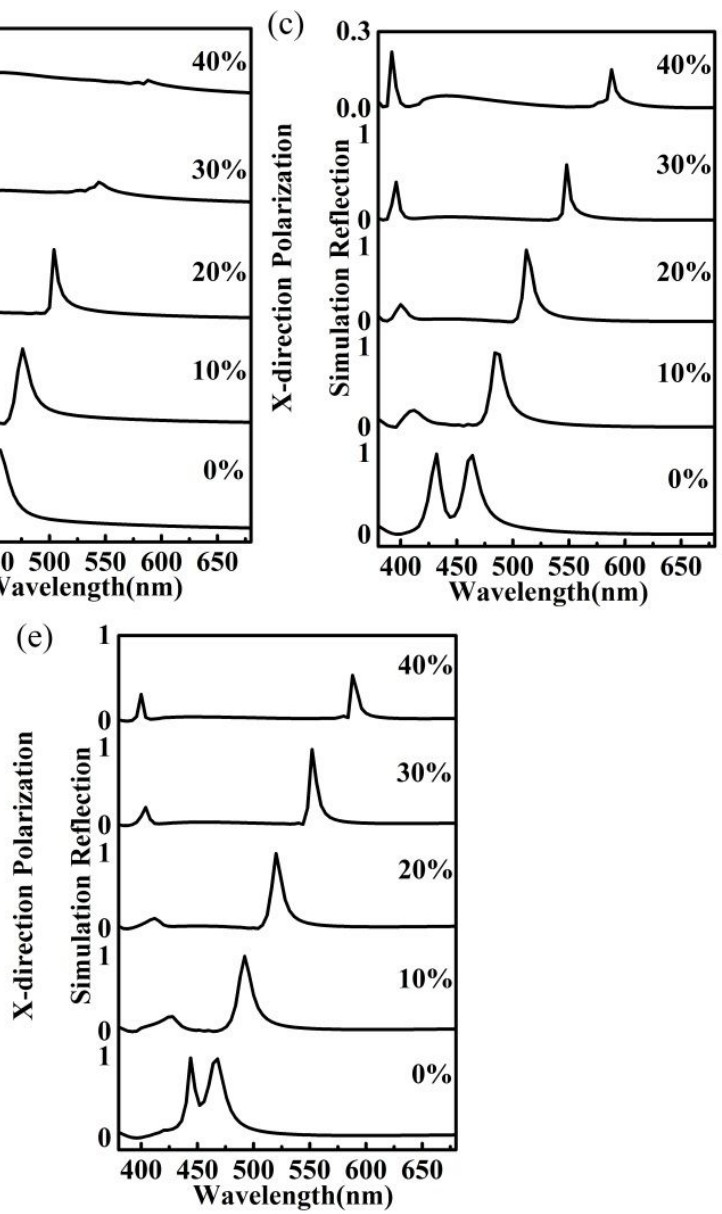

Figure S7. The numerically calculated reflection spectrum with different filling factor in x-polarization. (a) The reflection spectrum corresponds to different stretching amounts in filling factor is $0 \%$. (b) (c) (d) (e) are the corresponding reflection spectrums with filling factor from $25 \%, 50 \%, 75 \%$ to $100 \%$.

When the filling factor is lower than $50 \%$, the wavelength shift for x-polarization and y-polarization light under strain is quite different from each other. For $\mathrm{x}$-polarization light, the wavelength shift for $\mathrm{x}$-polarization light is much faster than y-polarization. However, when the filling factor of PDMS is above $50 \%$, the wavelength shift for $\mathrm{x}$-polarization and $\mathrm{y}$-polarization light under strain is consistent to each other. 
However, the filling factor is confirmed as $100 \%$ by the SEM images of the experiments. That is to say, the PDMS can be filled into the gap between the united cells. It can be seen from the simulation results that the effect of x-polarization or y-polarization is most obvious when the filling factor is $100 \%$. The saturation of the color seen in the experiment is the highest. With the stretching of the substrate, the mechanism of color change is most appropriate with theoretical analysis.
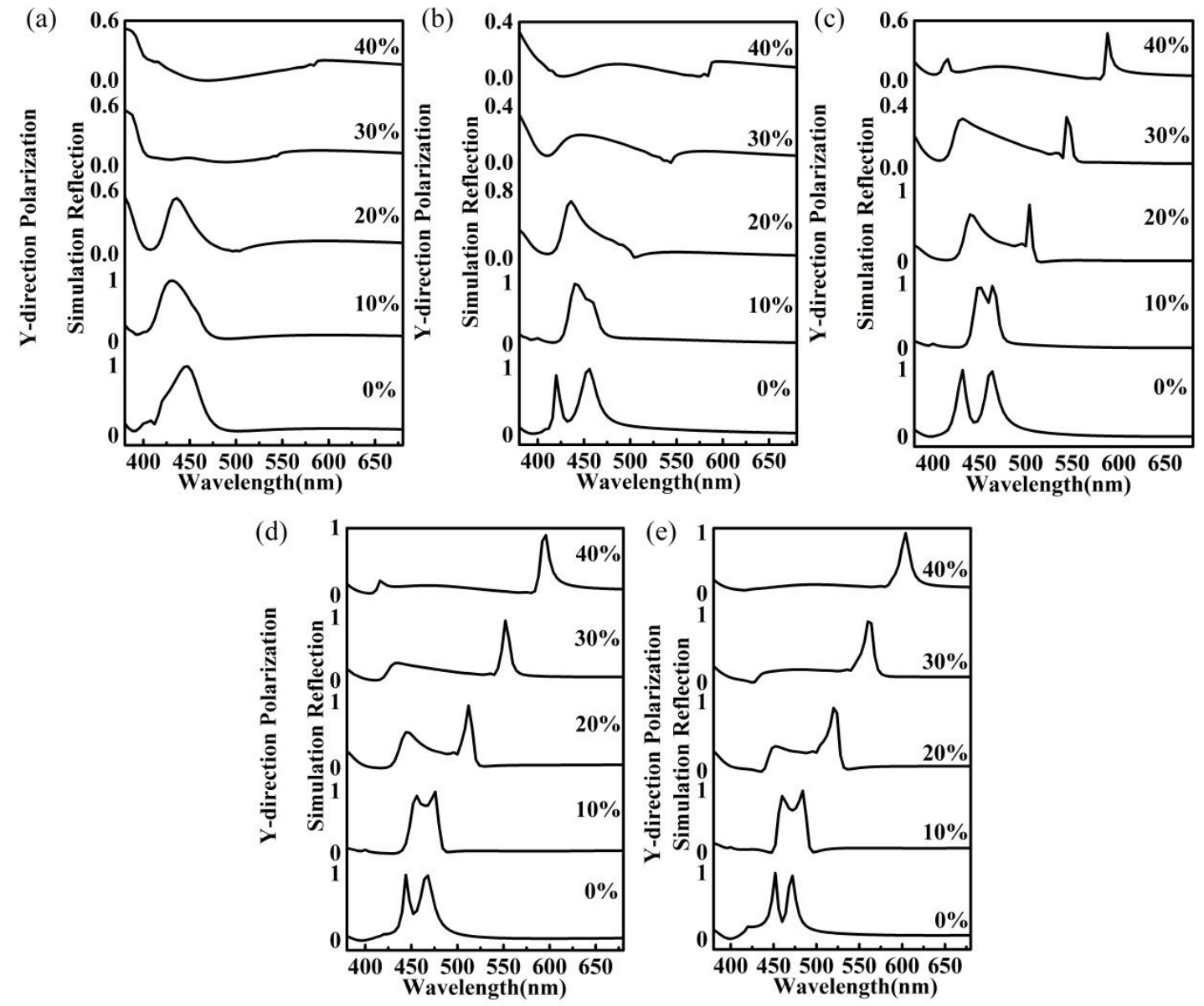

Figure S8. The numerically calculated reflection spectrum with different filling factor in y-polarization. (a) The reflection spectrum corresponds to different stretching amounts in filling factor is $0 \%$. (b) (c) (d) (e) are the corresponding reflection spectrums with filling factor from $25 \%, 50 \%, 75 \%$ to $100 \%$. 
After transferring the patterns to the PDMS substrate, the filling factor is $100 \%$. We numerically simulated the reflection spectrums of the PDMS filling factors with $50 \%, 75 \%$ and $100 \%$ (as Figure S7 and Figure S8 in supplementary materials). During change of strain from 0 to $40 \%$, the corresponding electric dipole and magnetic dipole shift under different working mechanisms. Summarize the wavelength of the electric and magnetic dipoles resonance in each case and compare with the experiment results. Analysis the results of Figure S9, the shift of reflectivity peaks and variation trend for $\mathrm{x}$-polarization and $\mathrm{y}$-polarization is almost synchronous and matching the simulation results for filling factor of $100 \%$ very well.

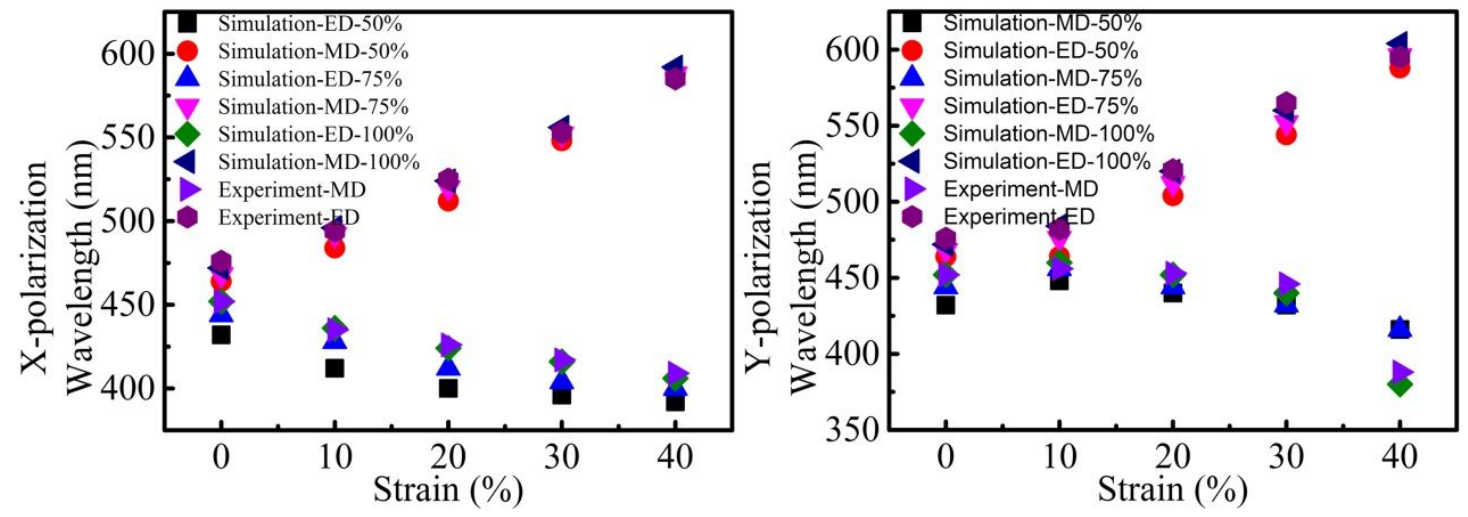

Figure S9. Compare the simulation and experiment resonance wavelength with different filling factor in different polarization.

In Figure S10 and Figure S11 are the shift behavior of two $\mathrm{TiO}_{2}$ metasurfaces with different geometries of $p_{1}=300 \mathrm{~nm}, w_{1}=230 \mathrm{~nm}$ and $p_{2}=320 \mathrm{~nm}, w_{2}=210 \mathrm{~nm}$. The initial reflection peaks are close to each other around $470 \mathrm{~nm}$ for both of samples. As a result, both of the metasurfaces show blue color in bright field and are hard to distinguished from each other. However, due to the difference of the gap distance the 
x-polarized MD resonance shift will experience different speed with the same stretching along $\mathrm{x}$-direction, and the metasurface with larger $g$ sample has a faster shift speed. Then as the amount of stretch increases, the spectrum and color difference between these two metasurface becomes larger and larger. When stretch of $30 \%$ is applied, the x-polarized MD resonance of metasurface with larger gap of $110 \mathrm{~nm}$ will move to around $580 \mathrm{~nm}$. And the x-polarized MD resonance of metasurface with smaller gap of $80 \mathrm{~nm}$ will move to the wavelength of $565 \mathrm{~nm}$, slightly slower than that for metasurface with larger gap.
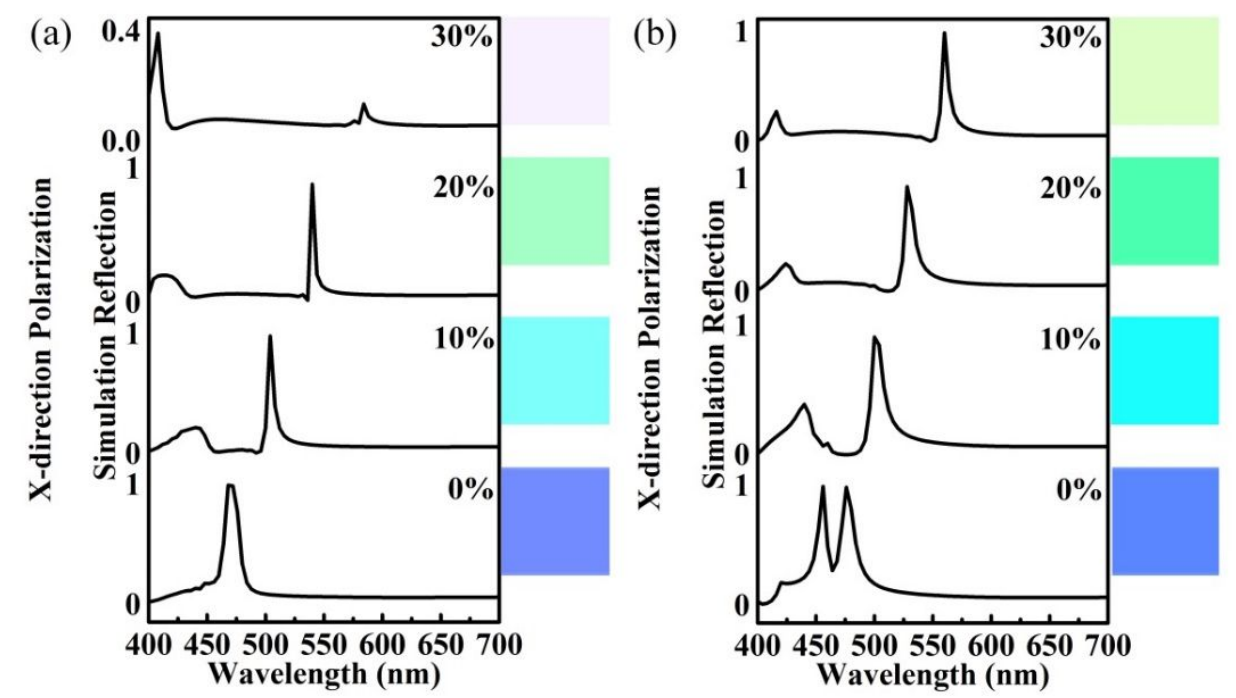

Figure S10. The reflection spectrums of different structural parameters in x-polarization. (a) The reflection spectrum of $p=320 \mathrm{~nm}, w=210 \mathrm{~nm}$ with stretch from $0 \%, 10 \%, 20 \%$ to $30 \%$. (b) The reflection spectrum of $p=300 \mathrm{~nm}, w=230 \mathrm{~nm}$ with stretch from $0 \%, 10 \%, 20 \%$ to $30 \%$.

For y-polarized ED resonance also has the same shift rule as the x-polarized MD resonance. When the amount of stretch is $30 \%$, the structure with larger gap has 
turned red, with the corresponding peak position moving to about $600 \mathrm{~nm}$, And sample with smaller gap just has turned to green, with the corresponding peak moving to $560 \mathrm{~nm}$.
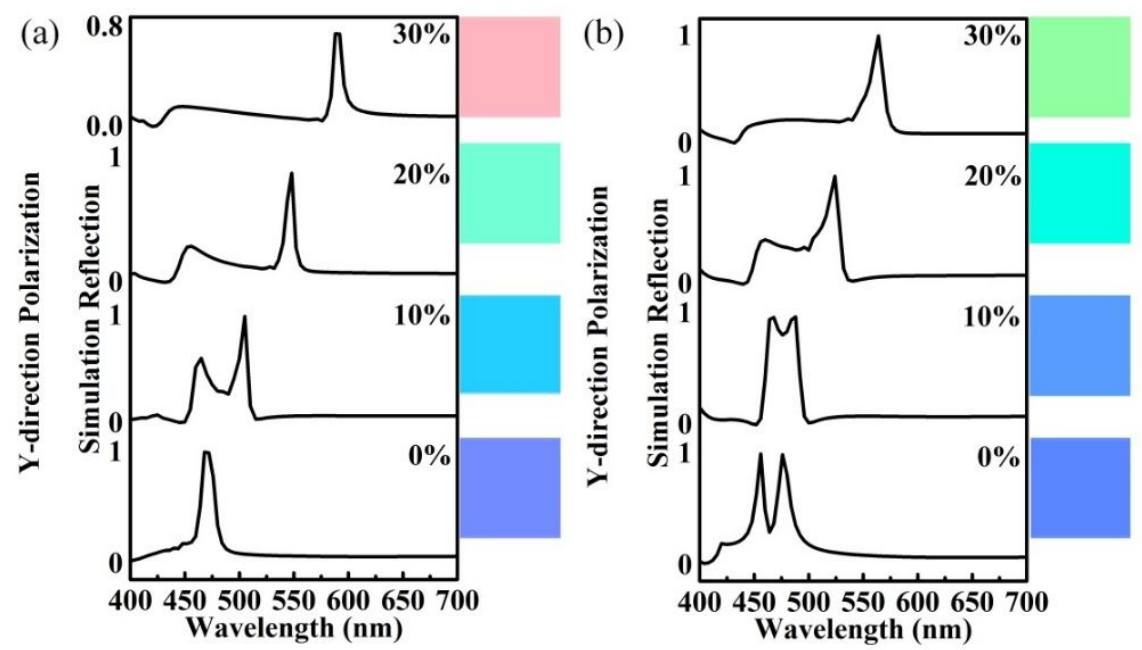

Figure S11. The reflection spectrums of different structural parameters in y-polarization. (a) The reflection spectrum of $p=320 \mathrm{~nm}, w=210 \mathrm{~nm}$ with stretch from $0 \%, 10 \%, 20 \%$ to $30 \%$. (b) The reflection spectrum of $p=300 \mathrm{~nm}, w=230 \mathrm{~nm}$ with stretch from $0 \%, 10 \%, 20 \%$ to $30 \%$.

The spatial resolution is an important parameter color nano-printing. Herein, we have simulated the reflection spectra of $\mathrm{TiO}_{2}$ nanoparticle arrays with different number of periods from infinity to $16 * 16,8 * 8$, and $4 * 4$. Here the structural parameters are kept as $w=230 \mathrm{~nm}$ and $p=300 \mathrm{~nm}$. All the results are summarized in Figure S12 below. With the decrease of period number, the blue structural colors are well kept to $8 * 8$ or even to $4 * 4$. When $20 \%$ strain has been applied onto the metasurface, all the colors are changed to green colors as well. In this sense, we know that the colors are well preserved in small pixels such as $4 * 4$. The size for $4 * 4$ pixel is 
$1.2 \mu \mathrm{m}$, giving a spatial resolution of $16000 \mathrm{dpi}$. This is orders of magnitude larger than the conventional pigments.
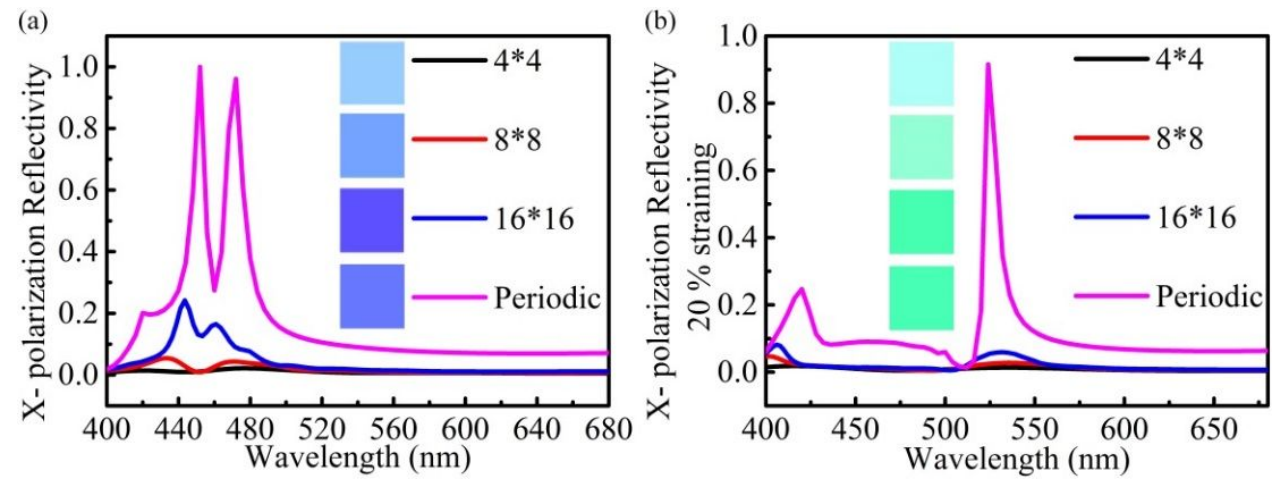

Figure S12. Simulated reflection spectra for different array and corresponding colors.

(a) X-polarization reflectivity in initial. (b) X-polarization reflectivity with the stretching amount $20 \%$.

The experimental results are lower and broader than the numerical calculations is mainly because of the un-avoidable roughness during the nanofabrication process. As the SEM images depicted in Figure 3A, the edges of $\mathrm{TiO}_{2}$ become very rough during the after the nanofabrication process. Such roughness produces very strong scattering loss and results in lower reflectivity peak. To verify the effect of loss, we have introduced an additional loss to the $\mathrm{TiO}_{2}$ material and simulated the reflectivity spectrum. As shown in Figure S13 (a) and (b), we can see the simulated spectra match the experimental results much better. 

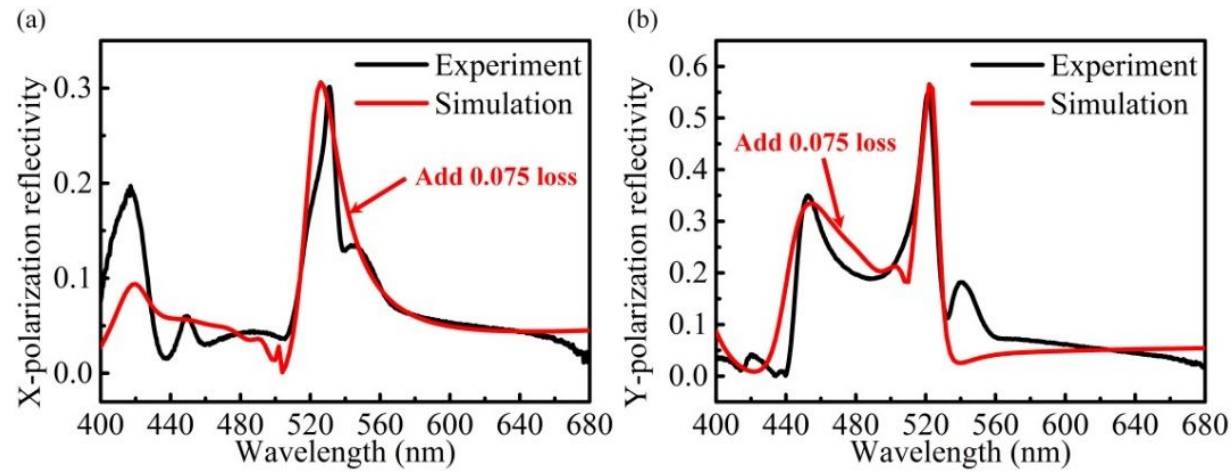

Figure S13. Experiment and simulation reflection spectrum. (a) and (b) X and Y polarization reflection spectrum with the numerical fitting results.

Figure S14 shows the setup for optical reflection characterization. The sample is hold onto two linear transition lines with both of resolution of $10 \mu \mathrm{m}$ under a home-made microscope. The characteristics of the reflection spectra were took under the normal incidence with a spectrometer. The bright-field microscopy images were taken under an optical microscope (ZEISS, Axio Scope AI) with Canon EOS 600D.

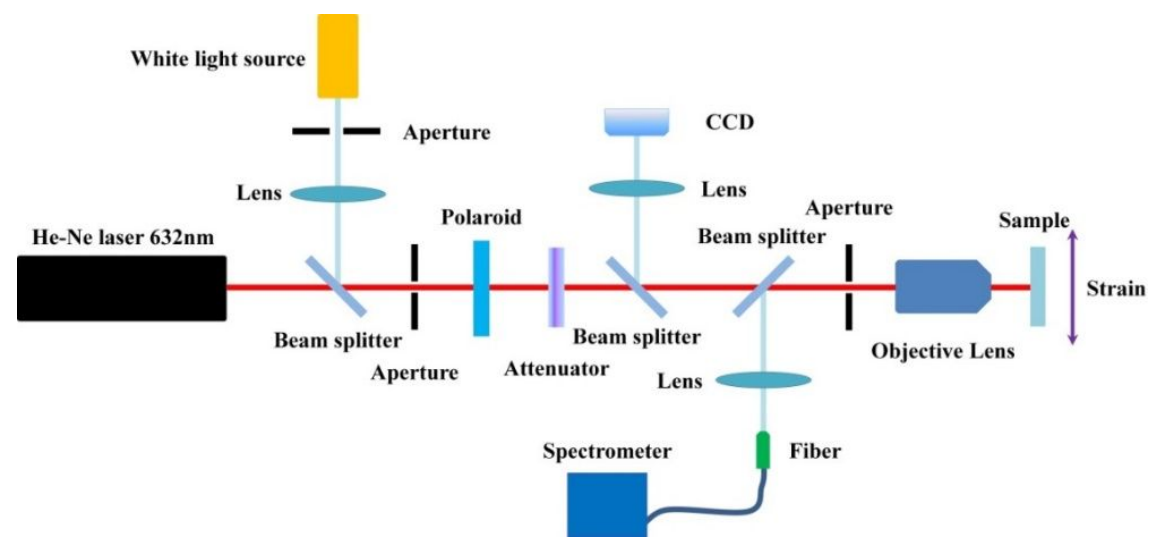

Figure S14. Schematic picture of the setup for optical characterization

Figure S15 is a physical diagram of our self-made fixture. It fixed both ends of the sample with two clips. The sample is stretched by rotating the spiral ruler, and the amount of stretching of the sample is accurately controlled with resolution of $10 \mu \mathrm{m}$. 


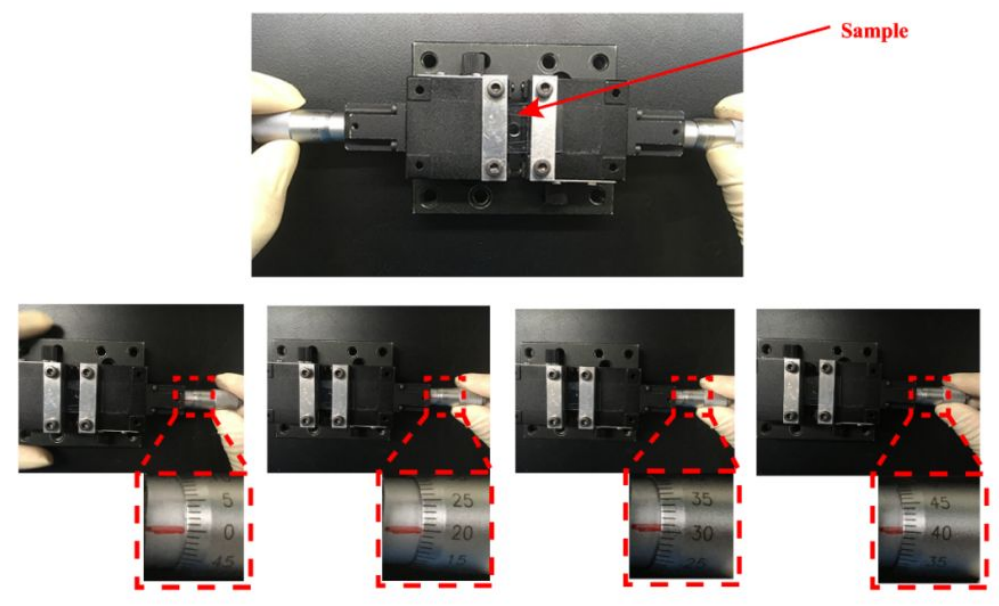

Figure S15. The physical diagram of our self-made fixture

The fabrication process of the $\mathrm{TiO}_{2}$ metasurfaces imbedded in PDMS is discussed in Methods. An epoxy-based negative photoresist (SU-8) with thickness of $1 \mu \mathrm{m}$ is spin-coated onto the copper foil substrate in order to decrease the surface roughness of the copper foil. The surface roughness of the copper foil substrate is measured using atomic force miscopy (AFM). Before the SU-8 is spin-coated onto the copper foil, the surface is extremely rough with the RMS number as large as $119 \mathrm{~nm}$, indicating the simple copper foil substrates is not suitable to work as sacrificial substrate. Spin-coating the SU-8 layer with thickness of $1 \mu \mathrm{m}$ on top of copper foil layer will decrease the surface roughness to as small as $3.27 \mathrm{~nm}$, which is smooth enough for structure fabrication. Further deposition of sacrificial copper layer with thickness of $150 \mathrm{~nm}$ doesn't affect the surface roughness by keeping the surface roughness around $3.12 \mathrm{~nm}$. Finally, we obtain a $\mathrm{Cu} / \mathrm{Su}-8 / \mathrm{Cu}$ substrate will can work as a sacrificial substrate to fabricate and transfer the $\mathrm{TiO}_{2}$ metasurface. 


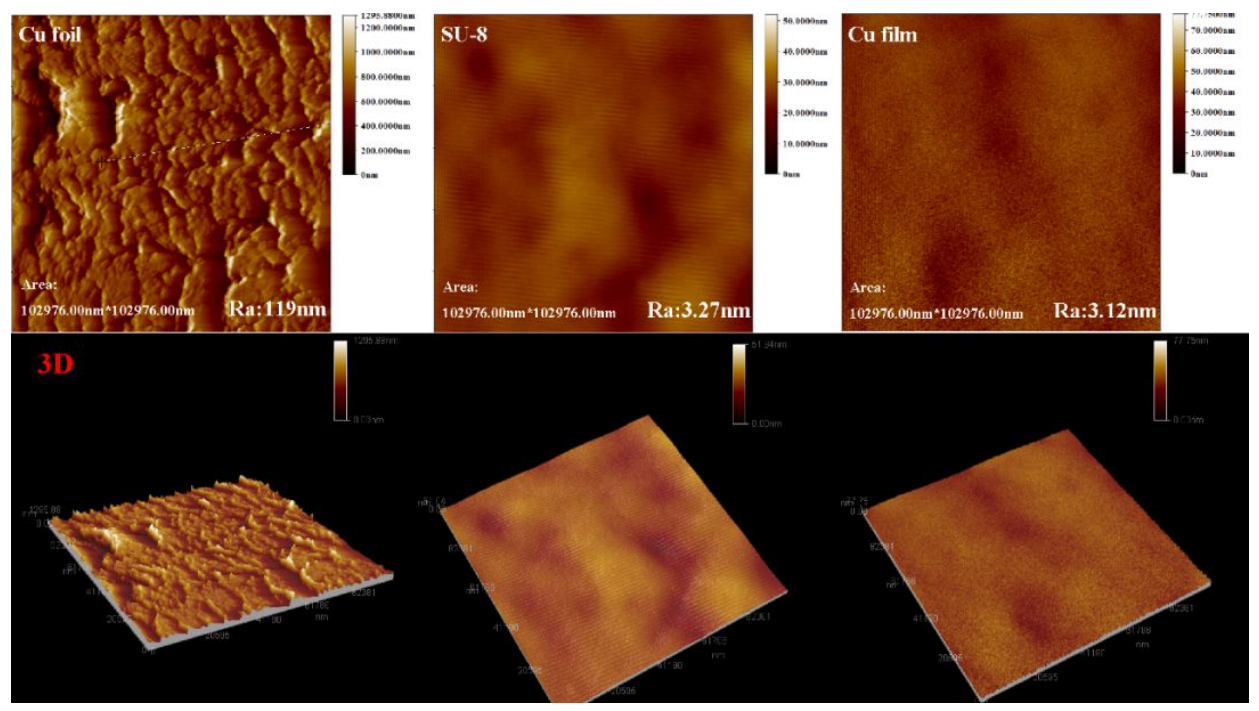

Figure S16. The surface roughness of the copper foil before and after spin-coating the Su-8, and after deposition additional $150 \mathrm{~nm}$ copper layer.

We have characterized the surface roughness and dispersion curve of our $\mathrm{TiO}_{2}$ film. The surface roughness of $\mathrm{TiO}_{2}$ film is measured by atomic force microscopy (AFM) and the result is shown in Figure S17. The profile roughness parameter Sq is around $1.0 \mathrm{~nm}$. The surface roughness is far below the wavelength and indicates that the film prepared by electron beam evaporation has very smooth surface.
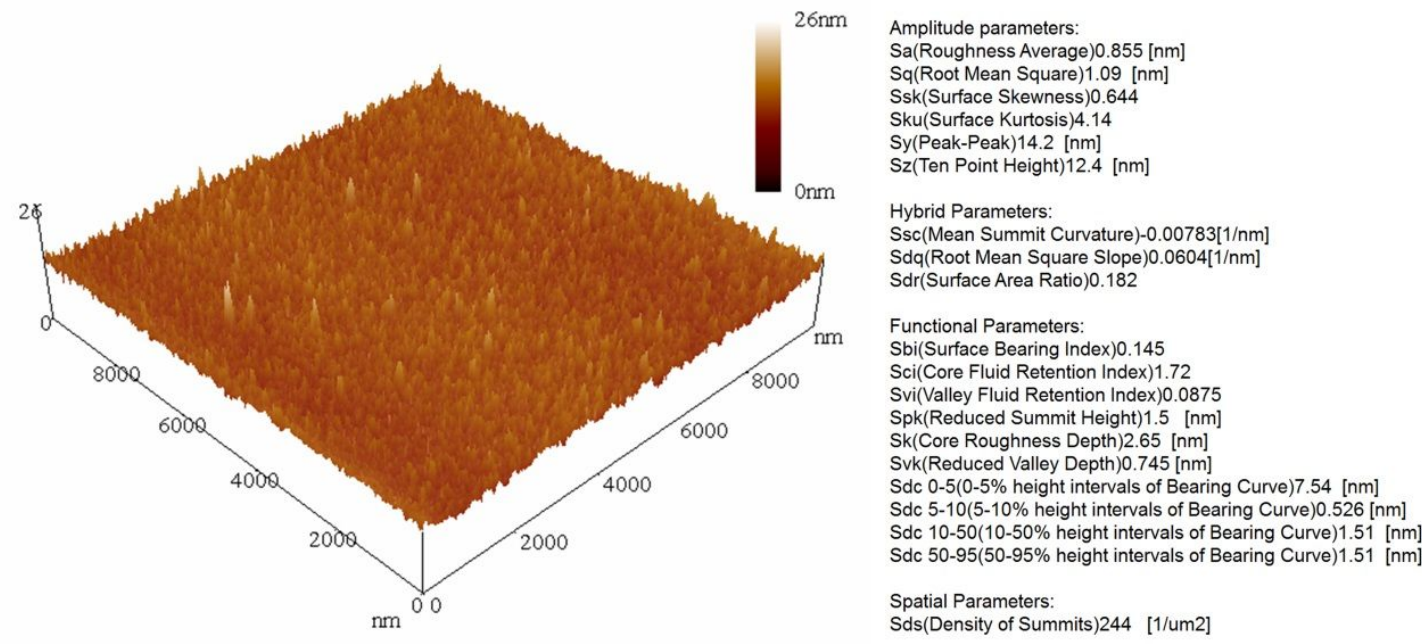

Figure S17. The AFM image of the $\mathrm{TiO}_{2}$ film. 
The optical constants of $\mathrm{TiO}_{2}$ thin film are also characterized by the ellipsometer (J.A.Woollam-M2000UI), and the values of $\mathrm{n}$ and $\mathrm{k}$ are finally obtained through the model fitting of the data. As shown in Figure S18, the real part of the refractive index n stays above 2.2 thorough the entire visible spectrum from $400 \mathrm{~nm}$ to $750 \mathrm{~nm}$, whereas the imagery part of the refractive index $\mathrm{k}$ stays around zero. The high real part of refractive index and negligibly small loss ensure that the deposited $\mathrm{TiO}_{2}$ film can be a good optical material to fabricate $\mathrm{TiO}_{2}$ metasurface.

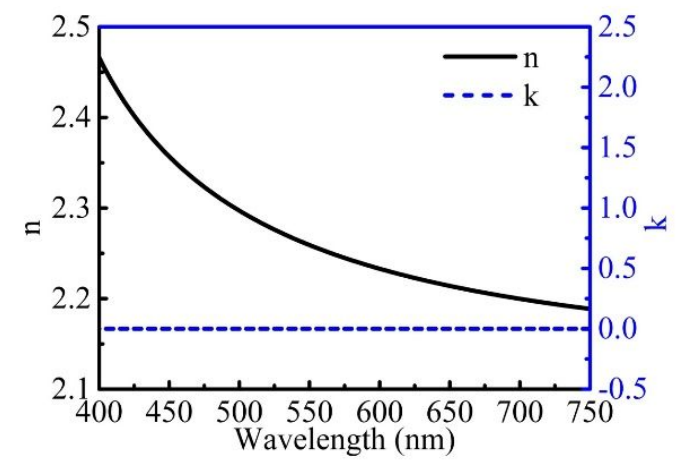

Figure S18. The refractive index (black solid line) and light extinction coefficient (blue dashed line) of $\mathrm{TiO}_{2}$ thin film.

Figure S19 is the experimental reflectance spectra for the x-polarization light after 100 cycles at different stretch varying from $0 \%$ to $50 \%$. The reflection wavelength, the resonance shape and intensity are well kept, conformed the high repeatability of the stretchable metasurface. 
(a)

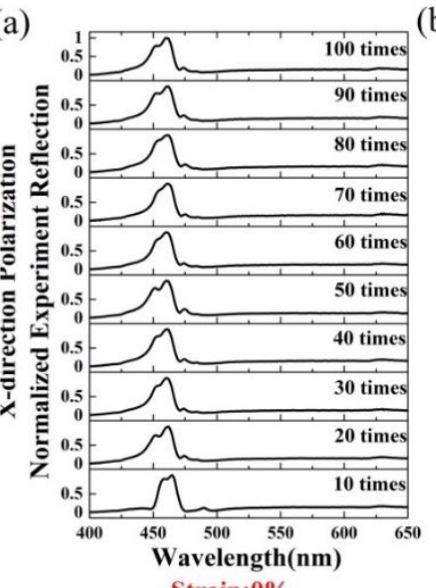

(d)

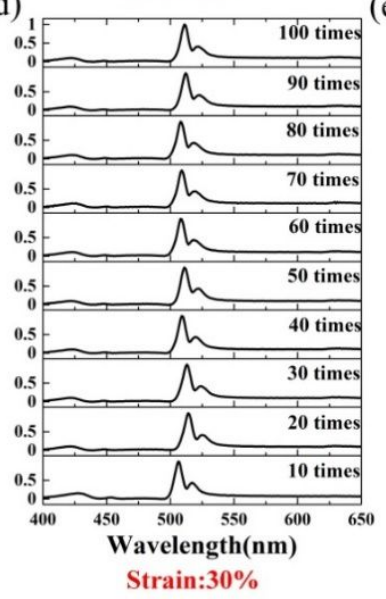

(b)

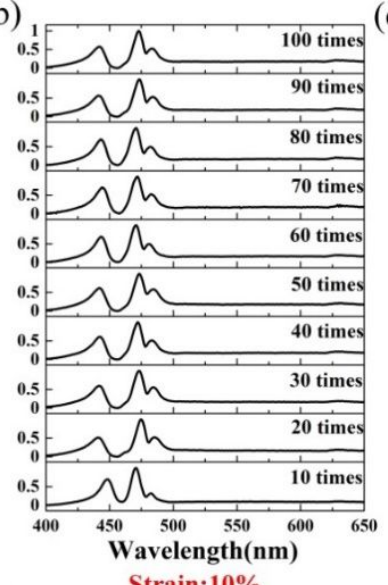

(e)

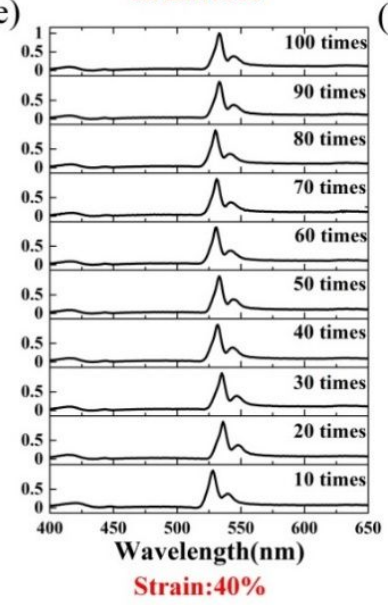

(c)

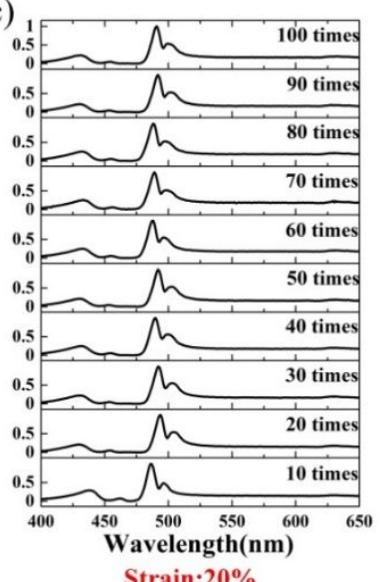

(f)

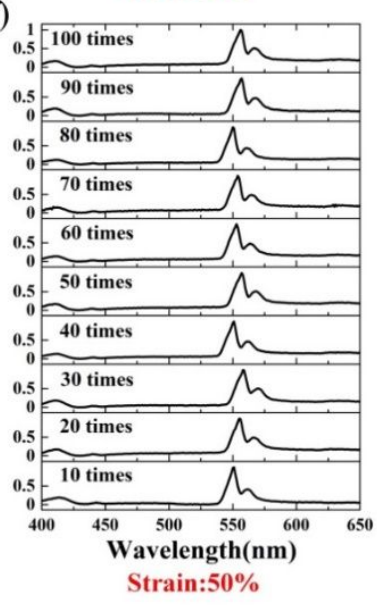

Figure S19. The reflection spectrums of different strain in x-polarization and cycled

100 times. (a) The reflection spectrums with the stretching $0 \%$ to cycle 100 times. (b)

(c) (d) (e) (f) are the corresponding reflection spectrums with strain $10 \%, 20 \%, 30 \%$,

$$
40 \%, 50 \% \text {. }
$$

Figure S20 is the experimental reflectance spectra of y-polarization light after 100 cycles also at different stretch amounts from $0 \%$ to $50 \%$. The results are similar to those for the x-polarization, and reflection wavelength, the resonance shape and intensity have no variation within the error range. This stretching cycle experiment demonstrates the stability of the substrate PDMS and nanostructures, promising real applications in the future. 
(a)

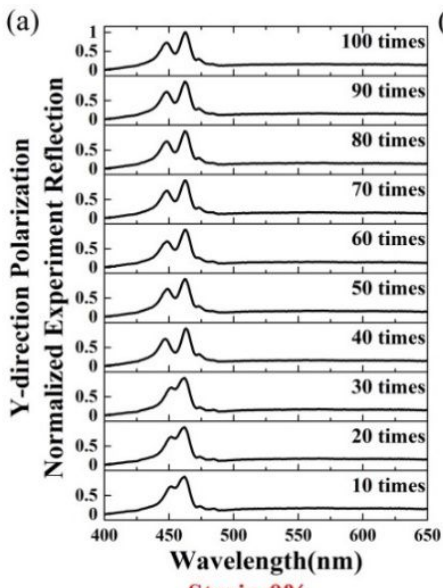

(d)

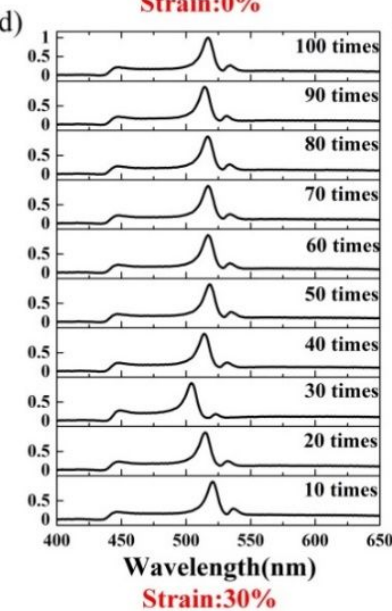

(b)

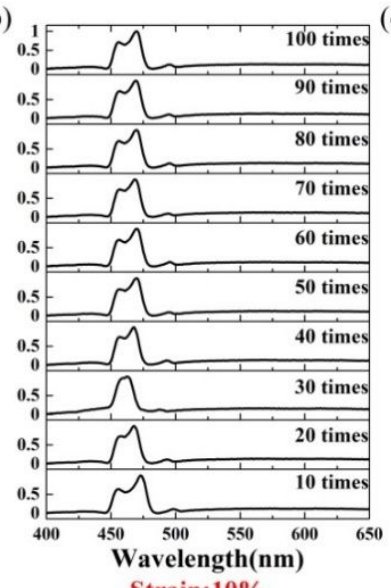

(e)

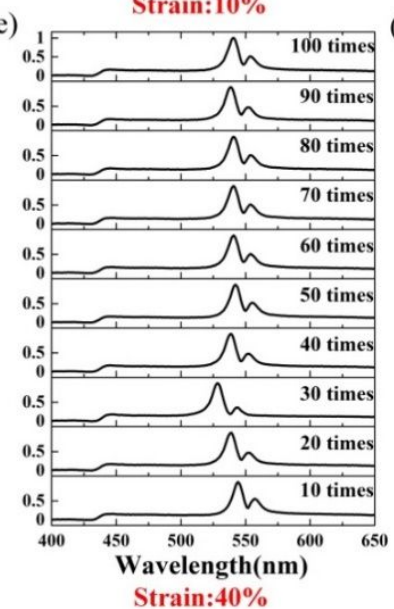

(c)

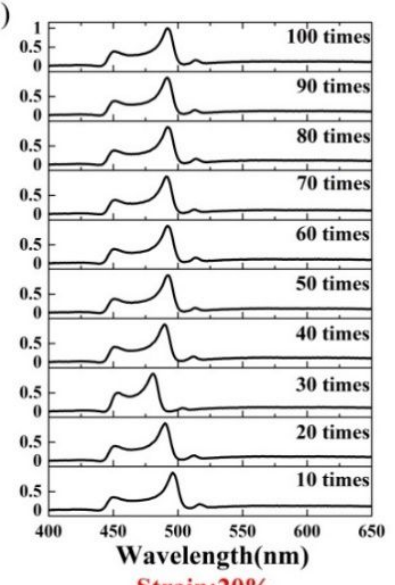

(f)

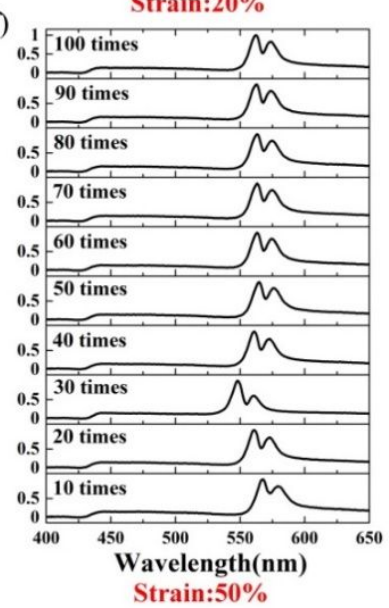

Figure S20. The reflection spectrums of different strain in y-polarization and cycled

100 times. (a) The reflection spectrums with the stretching $0 \%$ to cycle 100 times. (b)

(c) (d) (e) (f) are the corresponding reflection spectrums with strain $10 \%, 20 \%, 30 \%$,

$$
40 \%, 50 \%
$$

Figure S21 shows the top-view high resolution scanning electron microscope (SEM) image of $\mathrm{TiO}_{2}$ metasurface based images of "NANO" after transfer to PDMS. It can be seen the $\mathrm{TiO}_{2}$ metasurface is still maintaining integrity after transformation to PDMS, which greatly ensures the performance of the device. However the microscopic structural unit of nanostructure is almost indiscernible owing to the 
PDMS layer around surfaces. Furthermore, the PDMS has filled into the gap between the unit cells, indicating that the filling factor is $100 \%$.

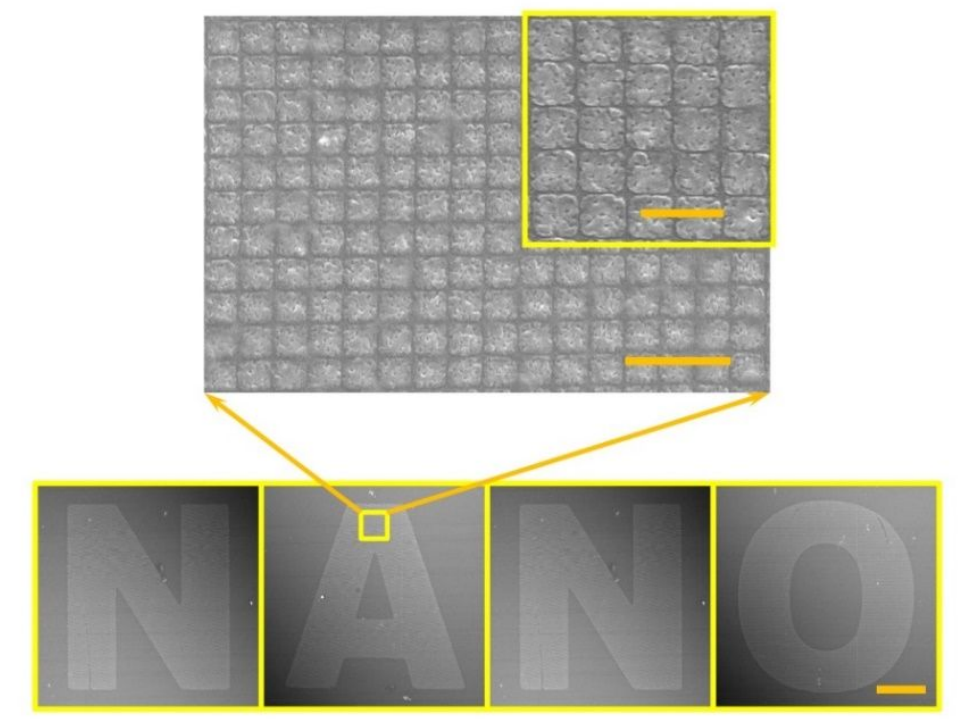

Figure S21. SEM images of the "NANO" after transformation to PDMS. The scale bar is $50 \mu \mathrm{m}, 1 \mu \mathrm{m}$ and $500 \mathrm{~nm}$ respectively.

In our experiment, the sample is mounted onto two three-dimensional translation stages with a resolution of $10 \mu \mathrm{m}$. Considering the sample size $(2 \mathrm{~cm} * 2 \mathrm{~cm})$, the accuracy and resolution of stretch is $5 \%$. In consideration of the excellent elasticity and uniformity of PDMS, the macroscopic stretching amount is also suitable at the microscopic level during the stretching process of PDMS. In other words, the total tension of PDMS substrate can represent the change in the period of structural unit cells at the microscopy level, which is $5 \%$. The dimensions of the structural can be expressed as $\mathrm{x}$-polarization $p_{\mathrm{x}}$ and $\mathrm{y}$-polarization $p_{\mathrm{y}}$ :

$$
p_{x}=p_{0}(1+t \times 100 \%)
$$




$$
p_{y}=p_{0}\left(1-\frac{t}{2} \times 100 \%\right)
$$

Where, $t$ is the strain value, $p_{0}=300 \mathrm{~nm}$ is the initial period. As a result, increase of periodicity along the x-direction is $15 \mathrm{~nm}$, whereas the decrease of periodicity along the y-direction is around $7.5 \mathrm{~nm}$. To confirm this information, we have measured the reflection spectra with a very small strain value of 5\% (see Figure S22). The wavelength shift matches the numerical calculation with the period change of 15 $\mathrm{nm}(\mathrm{x}$-direction) and $7.5 \mathrm{~nm}$ (y-direction) very well. Therefore, we can confirm that the precision and accuracy of the stretchable metasurface is $5 \%$.
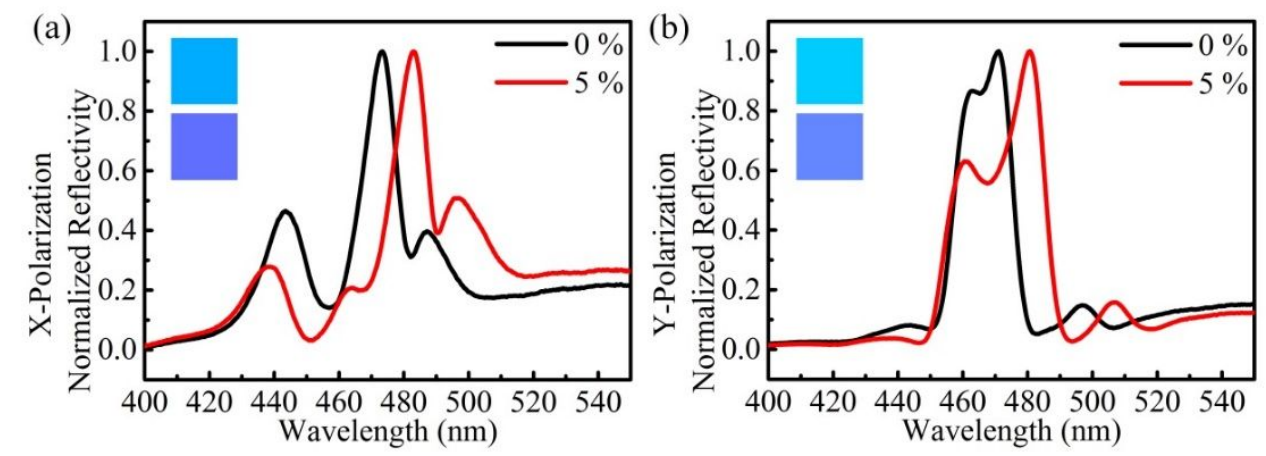

Figure S22. The measured reflection spectra of the $\mathrm{TiO}_{2}$ metasurface for x-polarized and y-polarized light at different strains. 